\title{
Traditional Chinese medicine Tongxinluo stimulates coronary collateral growth in a pig myocardial infarction model
}

\author{
Gao-Feng Wang ${ }^{1,2, \#}$, Sen Ye ${ }^{1, \#}$, Jian-Guo Cui ${ }^{3, \#}$, Naiquan Yang ${ }^{2, \#}, \mathrm{Ke} \mathrm{Xu}^{1}$, Shu-Hua Zhang ${ }^{4}$ and Chun-Jian $\mathrm{Li}^{1 *}$ \\ ${ }^{1}$ Department of Cardiology, First Affiliated Hospital of Nanjing Medical University, Nanjing 210029, China \\ ${ }^{2}$ Department of Cardiology, Huai'an Hospital affiliated to Xuzhou Medical College and Huai'an Second People's Hospital, Huaian 223002, China \\ ${ }^{3}$ Department of Gerontology, Affiliated Hospital of Binzhou Medical College, Binzhou 256603, China \\ ${ }^{4}$ Department of Cardiology, Second People's Hospital of Lianyungang, Lianyungang, 222000, China \\ \# These authors contributed equally to this work.
}

\begin{abstract}
Coronary collaterals were reported improving symptoms as well as prognosis for patients with coronary artery disease. This study aimed to investigate whether traditional Chinese medicine Tongxinluo could stimulate coronary collateral growth in a pig acute myocardial infarction (AMI) model. The AMI model was established by surgically ligation of left anterior descending coronary artery (LAD). Twenty-one Chinese Suzhong pigs were randomly divided into two control groups and two treatment groups taking Tongxinluo of $0.36 \mathrm{~g} \cdot \mathrm{kg}^{-1} \cdot \mathrm{d}^{-1}$ and $0.72 \mathrm{~g} \cdot \mathrm{kg}^{-1} \cdot \mathrm{d}^{-1}$ respectively. Coronary artery angiography $(\mathrm{CAG})$ and left ventriculography were performed to evaluate collaterals and cardiac function 1 month after the treatment, and the density of collateral capillaries of myocardial samples was also counted. The plasma levels of nitric oxide (NO) and endothelin-1 (ET-1) were serially determined. The density of capillaries was significantly higher in both low-dose and high-dose Tongxinluo groups than that in the negative control $(\mathrm{P}<0.01)$ and the positive control groups $(\mathrm{P}<0.05)$. Grade III collateral circulation was found in both treatment groups one month after LAD ligation, and the grades of collateral circulations were positively correlated to the Tongxinluo dosages $(\mathrm{P}=0.0191)$. The cardiac function, the plasma levels of NO and ET-1 showed no significant difference among the treatment and positive control groups. This study demonstrated that Tongxinluo stimulates coronary collateral growth in a pig AMI model.
\end{abstract}

\section{Introduction}

Coronary artery disease (CAD) is characterized by coronary artery stenosis or occlusion, which is presently the leading cause of death. Around 7.3 million people died from CAD every year accounting for approximately $13 \%$ of total death worldwide [1].

Percutaneous coronary intervention (PCI) and coronary artery bypass grafting $(\mathrm{CABG})$ are currently two regular revascularization strategies. However, both methods are unsuitable for patients with diffuse distal coronary atherosclerosis. An alternative treatment strategy to bridge this gap is to create coronary collaterals to improve the patients' symptoms as well as the prognosis [2,3]. Gene therapy has been promising to achieve biological bypass effect [4]. However, it may take long time before this strategy is developed mature enough to be widely applied in clinical practice [5].

Tongxinluo is a mixture of tranditional Chinese herbal medicine including Radix ginseng, Scorpio, Hirudo, Eupolyphaga seu steleophage, Scolopendra, Periostracum cicadae, Radix paeoniae rubra, and Borneolum syntheticum, which has been widely used to treat patients with coronary artery diseases in China [6]. Former studies reported that Tongxinluo had effects of lowering lipids [7], eliminating inflammation [8], stabilizing vulnerable plaques $[7,8]$, inhibiting ventricular remodeling [9], and improving endothelial function $[10,11]$. However, it is unclear whether Tongxinluo improves angiogenesis by promoting the growth of the coronary collaterals.
In this study, we established a pig acute myocardial infarction (AMI) model and investigated the biological bypass effect of the traditional Chinese medicine Tongxinluo.

\section{Materials and methods}

\section{Study subjects}

Twenty-one ordinary level Chinese Suzhong pigs, male or female, weighted $30 \pm 5.6 \mathrm{~kg}$, were recruited in the study. The experimental animals were bred in Jiangsu Academy of Agricultural Sciences and the certificate number of the animal breeder was S000103. Three of the subjects were randomly selected to compose the negative control group without receiving the model construction and were fed with placebo. Three pigs died during the establishment of the AMI model, and the remaining 15 subjects were randomly assigned into the following three groups after undergoing successful coronary artery ligation: 1) positive control group ( $\mathrm{n}=5)$, fed with placebo for one month; 2) Low-dose Tongxinluo group $(n=5)$, fed with Tongxinluo at a dose of $0.36 \mathrm{~g} . \mathrm{kg}$

Correspondence to: Chun-Jian Li, Department of Cardiology, First Affiliated Hospital of Nanjing Medical University, 300, Guangzhou Road, Nanjing 210029, China, Tel: 86-25-68136018, Fax: 86-25-83674380,E-mail: lijay@njmu.edu.cn

Key words: tongxinluo, acute myocardial infarction, coronary collateral circulation Received: June 03, 2017; Accepted: June 23, 2017; Published: June 26, 2017 
${ }^{1} \cdot \mathrm{d}^{-1}$ for one month , and 3) high-dose Tongxinluo group $(\mathrm{n}=5)$, fed with Tongxinluo at a dose of $0.72 \mathrm{~g} \cdot \mathrm{kg}^{-1} \cdot \mathrm{d}^{-1}$ for one month. Tongxinluo microfine powder was provided by Shijiazhuang Yiling Pharmaceutic Co. Ltd., Hebei, China. The experiments were conducted according to the National Research Council's guidelines. The study protocol was approved by the Laboratory Animal Ethics Committee, First Affiliated Hospital of Nanjing Medical University, China.

\section{Establishment of the AMI model}

Animals were anesthetized with ketamine, intubated orally for artificial respiration and maintained with fentanyl. After regular preparation, a limited left thoracotomy was performed through the third and fourth intercostal space. Subsequently, the pericardium was gently opened, and the left anterior descending coronary artery (LAD) was then dissected out, after which mid-LAD was ligated exactly distal to the origin of the first diagonal branch with \#4 silk suture to ensure a total occlusion of the artery. The chest was finally closed, and the pigs were allowed to recover under intensive care. The electrocardiography (ECG) was recorded prior to and $4 \mathrm{~h}$ after LAD ligation.

\section{Coronary angiography}

Coronary angiography (CAG) was performed for all pigs $4 \mathrm{~h}$ after the establishment of AMI model to ensure occlusion of the LAD. $6 \mathrm{~F}$ arterial sheath was inserted in the right femoral artery. Under the X-ray fluoroscopy, the JL-3.5 and JR-3.5 catheters were advanced to engage the left and right coronary artery ostium respectively, then $\mathrm{CAG}$ was performed and all the images were recorded. Another CAG was performed one month later to evaluate the condition of the ligated coronary artery and the associated collaterals. The left ventriculography was also performed during the second CAG and the left ventricular ejection fraction was determined using the commercial Centricity Cardiology CA 1,000 software v1.0 (General Electric Medical Systems, USA) [12]. The angiographic collaterals were classified into 4 grades depending on the presence and the filling of the LAD [13].

\section{Pathological study}

At the end of the experiment, all animals were euthanized. The hearts obtained from the pigs were cut into $3 \mathrm{~mm}$ short-axis sections from base to apex of the ventricules. The basal short axis slice was positioned just forward of the atrioventricular ring, and all subsequent sections were acquired in $3 \mathrm{~mm}$ thickness towards the apex. Two sections of the second upper and lower parts of ventricle were selected to perform immunohistochemical analysis.

Tissue samples taken from the two edges of the infarction area of the two sections were fixed and embedded in paraffin, then cut into 10 $\mu \mathrm{m}$-thick slices and reacted with VIII factor antibody. Capillaries of the samples were observed by an investigator who was blinded to the study protocol with an optical microscope and photomicrographs were taken using a digital image analysis system. The density of capillaries was evaluated by counting vessels in 3 randomly chosen high-power fields $(400 \times)$ in each slice and the density of capillaries were expressed as VIII factor ${ }^{+}$arterioles $/ \mathrm{mm}^{2}$ (the area of 400 high-power fields is equal to $0.152 \mathrm{~mm}^{2}$ ). The averages of each high-power field of both edges of the two sections were used for comparison.

The remaining sections were immersed in $2 \% 2,3$, 5-triphenyltetrazolium chloride (TTC) at $37^{\circ} \mathrm{C}$ for $15 \mathrm{~min}$. The normal myocardial tissue turned into red color and the infarct tissue turned into white after TTC staining. The infarction area was determined by Adobe Photo-Shop CS after taking a photograph and presented as the percentage of (myocardial infarction area / left ventricule area) $\times 100$.

\section{Determination of cardiac troponin-I (cTnI), plasma nitric oxide (NO) and endothelin-1(ET-1)}

Serum levels of cTnI was determined 4-6h after the LAD ligation by the method of "sandwich" ELISA described previously [14]. Plasma levels of NO and ET-1 were determined prior to the establishment of AMI model, 4-6h post-LAD ligation and during 1 month follow-up. Plasma levels of NO were measured by chemiluminescence using a highly sensitive NO measurement system (FES-450; Scholar-Tec Co., Ltd., Osaka, Japan). Plasma levels of ET-1 were measured by enzymelinked immunosorbent assay (ELISA; RIA2 method, BML, Tokyo, Japan).

\section{Statistical analysis}

Statistical analyses were carried out using Stata Statistical Software for Professionals (version 11, Stata Corp, College Station, Texas). Quantitative variables are expressed as means \pm SD. Comparison of continuous variables among multiple groups was performed by oneway analysis of variance (ANOVA). Fisher's exact test and spearman rank correlation were adopted to analyze the difference of the collateral circulations among different groups. $\mathrm{P}<0.05$ was considered to be statistically significant.

\section{Results}

\section{Evaluation of the AMI model}

ECG showed that the ST segment of the chest leads elevated in all pigs after the LAD ligation. CAG proved that LAD ligation was successfully performed with total occlusion in every established AMI model (Figure 1). The cTnI levels of all animals were $>0.1 \mathrm{ng} / \mathrm{ml}$ except 1 in positive control group and 1 in high-dose Tongxinluo group. One pig in the high-dose group died 25 days after the LAD ligation.

\section{Coronary collateral circulation}

Immunohistochemisty examination with VIII factor antibody showed that myocardial capillaries were stained in brown color (Figure 2). There were significantly more capillaries in the low-dose Tongxinluo group than in the negative control group $(651.32 \pm 197.37$ vs. $111.84 \pm$ $6.58, \mathrm{p}<0.01)$ and positive control group $(651.32 \pm 197.37$ vs. 289.47 $\pm 223.68, \mathrm{p}<0.05$ ) (Figure 3). Similarly, there were significantly more capillaries in the high-dose Tongxinluo group than in the negative control group $(585.53 \pm 125$ vs. $111.84 \pm 6.58, \mathrm{p}<0.01)$ and positive control group $(585.53 \pm 125$ vs. $289.47 \pm 223.68, \mathrm{p}<0.05)$ (Figure 3).

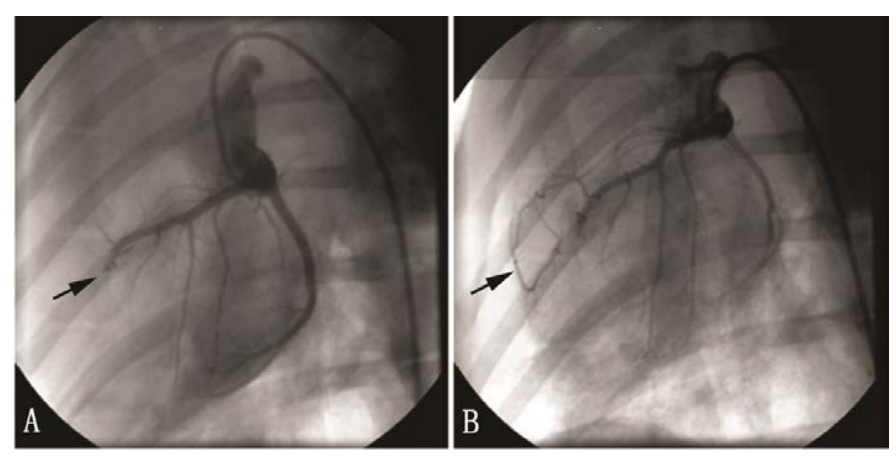

Figure 1. Coronary angiogram ofthe pig AMI model. A: arrow indicates proximal LAD totally occluded after LAD ligation. B: arrow indicates the collateral vessels formed and the distal LAD showed up although the proximal LAD still occluded1 month after the establishment of the AMI model. 


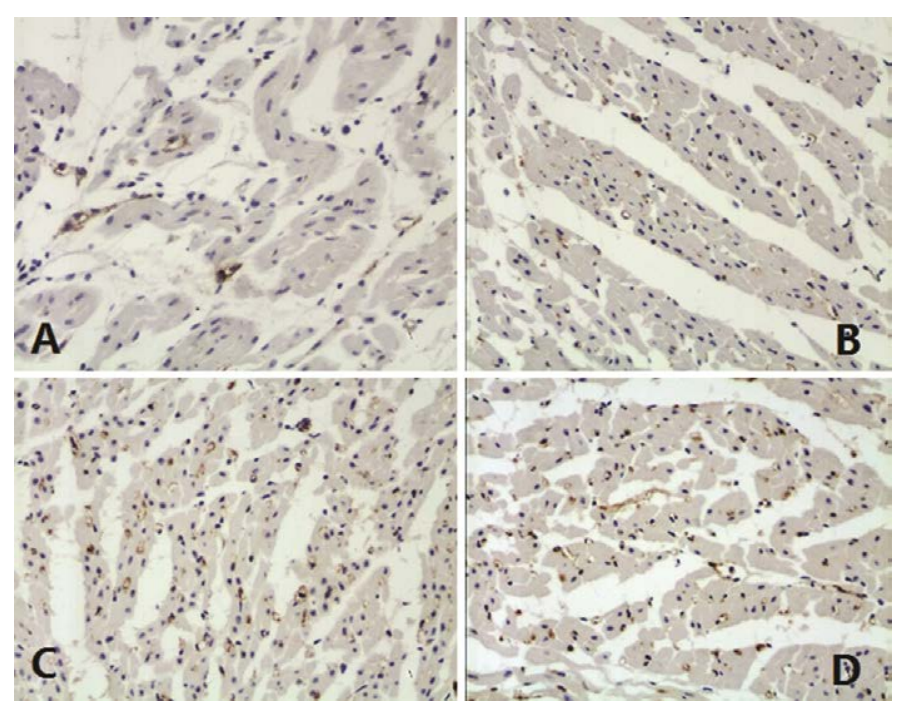

Figure 2. Capillaries of myocardium stained with VIII factor antibody. A, Negative control group; B, Positive control group; C, Low-dose tongxinluo group; D, High-dose tongxinluo group.

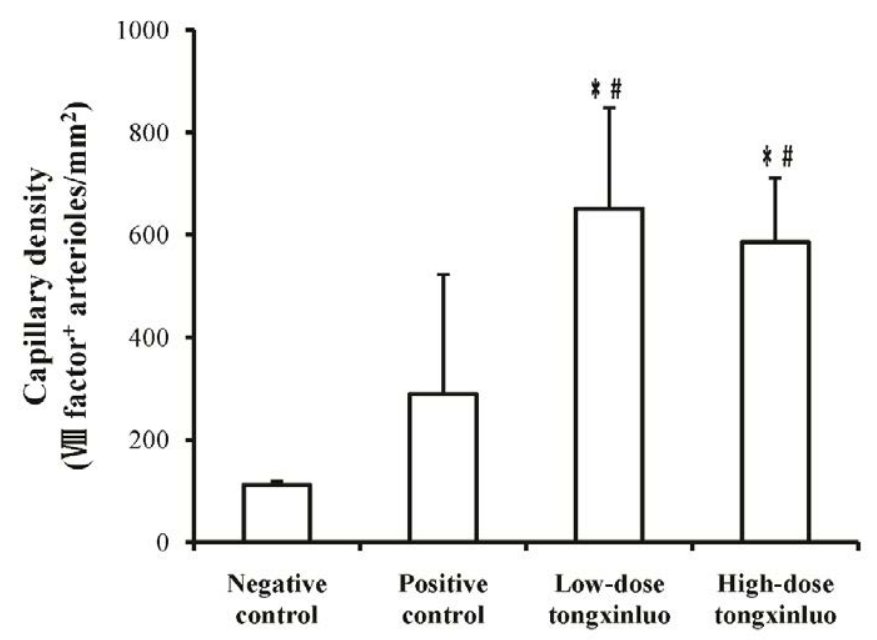

Figure 3. Capillary density in different groups. The collateral capillary vessel counts significantly increased in both the low-dose tongxinluo and the high-dose tongxinluo groups compared with the negative control group, and the positive control group. ${ }^{\text {" }} \mathrm{P}<0.01$ vs. negative control group. $\mathrm{P}<0.05 v s$. positive control group.

No difference of myocardial capillary density was found between the two treatment groups (Figure 3).

CAG showed that Grade III collateral circulation was found in 1 subject in the low-dose group, and 3 subjects in the high-dose group. Although the collateral circulation had no significant difference among the two treatment groups and the positive control group $(\mathrm{P}=0.110)$, the grades of collateral circulations were positively correlated to the Tongxinluo dosages $(\mathrm{P}=0.0191)$ (Table 1$)$.

\section{Myocardial infarction area and cardiac function}

The myocardial infarction areas of the positive control group, low-dose and high-dose Tongxinluo groups were $10.8 \pm 1.4 \%, 9.9 \pm$ $1.5 \%$, and $10.0 \pm 2.1 \%$ respectively. There was no significant difference among the three groups (Figure 4). Similarly, there was no significant difference of left ventricular ejection fraction (LVEF) among the positive control group, low-dose and high-dose Tongxinluo groups
Table 1. Rentrop score of the LAD collaterals. LAD, left anterior descending coronary artery; TXL, tongxinluo; "the LAD re-opened; *"the pig died.

\begin{tabular}{|l|c|c|c|c|c|}
\hline Subject & No.1 & No.2 & No.3 & No.4 & No.5 \\
\hline Postive control & 2 & $-*$ & 2 & 2 & 2 \\
\hline Low-dose TXL & 2 & 2 & 2 & 2 & 3 \\
\hline High-dose TXL & 2 & 3 & $-* *$ & 3 & 3 \\
\hline
\end{tabular}
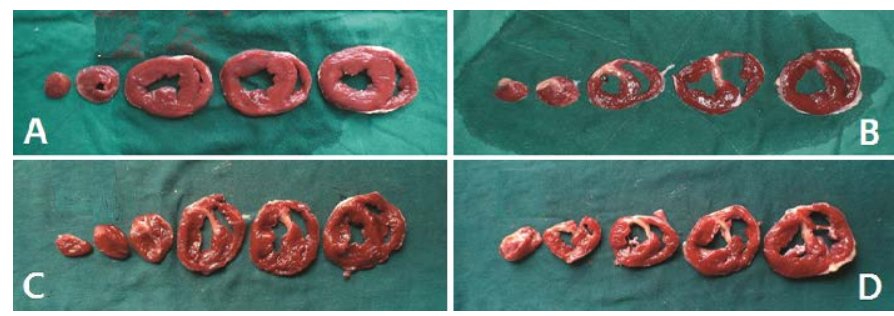

Figure 4. TTC staining of the myocardial infarctionA, Negative control group; B, Positive control group; C, Low-dose tongxinluo group; D, High-dose tongxinluo group. The normal myocardial tissue was stained in red and infarcttissue was stained in white. TTC, 2, 3, 5-triphenyltetrazolium chloride.

Table 2. Plasma levels of NO and ET-1. NO, nitric oxide; ET-1, endothelin-1; Baseline, before ligation of the coronary artery; LAD, left anterior descending coronary artery; ${ }^{*} \mathrm{P}=0.046$ vs. baseline; $\# \mathrm{P}=0.017$ vs. baseline.

\begin{tabular}{|c|c|c|c|}
\hline & Baseline & 4-6h post-LAD ligation & 1- \\
\hline Positive control & & & \\
\hline NO $(\mu \mathrm{mol} / \mathrm{L})$ & $112.7 \pm 56.0$ & $76.2 \pm 51.1$ & $143.2 \pm 123.4$ \\
\hline ET-1 $(\mathrm{pg} / \mathrm{ml})$ & $40.1 \pm 6.9$ & $39.0 \pm 15.6$ & $33.0 \pm 3.3$ \\
\hline Low-dose group & & & \\
\hline NO $(\mu \mathrm{mol} / \mathrm{L})$ & $116.6 \pm 68.4$ & $78.0 \pm 69.2$ & $70.1 \pm 44.8$ \\
\hline ET-1 $(\mathrm{pg} / \mathrm{ml})$ & $51.1 \pm 3.7$ & $39.2 \pm 20.1$ & $42.8 \pm 7.8^{*}$ \\
\hline High-dose group & & & \\
\hline NO $(\mu \mathrm{mol} / \mathrm{L})$ & $79.2 \pm 28.8$ & $54.7 \pm 22.0^{\sharp}$ & $57.6 \pm 15.7$ \\
\hline ET-1 $(\mathrm{pg} / \mathrm{ml})$ & $50.4 \pm 14.3$ & $70.5 \pm 35.5$ & $62.9 \pm 16.8$ \\
\hline
\end{tabular}

$(43.9 \pm 6.7 \%$ vs. $36.9 \pm 2.4 \%$ vs. $41.3 \pm 13.6 \%, \mathrm{p}>0.05)$.

\section{NO and ET-1 levels}

The serum level of NO significantly decreased $4-6 \mathrm{~h}$ after LAD ligation compared with the baseline level $(54.7 \pm 22.0 \mu \mathrm{mol} / \mathrm{L}$ vs. 79.2 $\pm 28.8 \mu \mathrm{mol} / \mathrm{L}, \mathrm{P}=0.017)$ in the high-dose Tongxinluo group, and the serum level of ET-1 significantly decreased at 1-month followup compared with the baseline level $(42.8 \pm 7.8 \mathrm{pg} / \mathrm{ml} v s .51 .1 \pm 3.7$ $\mathrm{pg} / \mathrm{ml}, \mathrm{P}=0.046)$ in the low-dose group. However, no significant differences of NO or ET-1 levels were found between the time points of post-LAD ligation and 1-month follow-up in either the positive control or treatment groups. In addition, no significant differences of NO and ET-1 were found among the two treatment groups and the positive control group at time points of baseline, post-LAD ligation and 1-month follow-up (Table 2).

\section{Discussion}

Tongxinluo Capsule is a traditional Chinese herbal compound which was developed by Shijiazhuang Yiling Pharmaceutic Co. Ltd., Hebei, China according to the general principle of unblocking the collateral by supplementing qi, resolving stasis or removing wind [15]. It has been approved in 1996 by the State Food and Drug Administration of China for treatment of angina pectoris and ischemic stroke. However, the pharmacodynamics of Tongxinluo has not been fully elucidated. In this study, we found that Tongxinluo superfine powder improves the myocardial capillary density at the edges of the infarction area, which 
might be associated with a better collateral circulation in the treatment groups. To the best of our knowledge, this is the first study reporting that traditional Chinese medicine Tongxinluo has the biological bypass effect for the occluded coronary arteries by immunohistochemical analysis as well as coronary artery angiography.

The mechanism of the angiogenesis effect of Tongxinluo is not clear. However, Bai et al. recently found that Tongxinluo treatment increased the expression of PI3K and phosphorylation of Akt as well as ERK, with an increased expression and DNA binding activity of HIF$1 \alpha$. HIF-1a can directly influence the expression of VEGF and eNOS $[16,17]$. VEGF, an endothelial-specific mitogen and survival factor, is one of the most potent angiogenic factors, and is a key factor for both angiogenesis and vasculogenesis [18] eNOS can be activated in phosphorylation of serine 1177 residue and this triggers migration and angiogenesis $[19,20]$.

ET-1 level rises acutely as early as several minutes after the onset of myocardial infarction $[21,22]$. The over-expression of endothelin-1 can inhibit the activity of endothelial NO synthase, and thereby decreasing NO level and bioavailability $[23,24]$. When the delicate balance between NO and ET-1 is disturbed, endothelial dysfunction may occur. Wu et al. found tongxinluo has a protective effect on vascular endothelium, for it could significantly increase the plasma NO level and decrease ET level in vascular endothelial dysfunction rats [25]. Dai et al. also showed that the levels of ET-1 and NO in endothelial dysfunction rats were up- and down-regulated respectively compared to healthy rats, and Tongxinluo could return these levels to the normal values [10]. In our study, we found that the plasma level of NO significantly decreased after LAD ligation in accordance with the previous studies. However, we did not find the effect of tongxinluo in decreasing ET-1 and increasing NO levels in this AMI pig model.

Bai et al. recently found that Tongxinluo can significantly improve the myocardial capillary density in rat myocardial infarction model [16]. However, immunohistochemical myocardial capillaries may not represent the functional coronary collateral circulation. In this study, we also found that the myocardial capillary density significantly increased in the treatment groups. Moreover, we found that the treatment groups tend to have more Grade III collaterals, which has not been reported previously. A well-developed coronary collateral circulation plays an important role in limiting infarct size, decrease the incidence of ventricular aneurysm formation, recovering cardiac function, preventing the future cardiovascular events, and improving survival [2,26-29]. In this study, the left ventriculography results showed that there was no significant difference between the treatment groups and the positive control group regarding the left ventricular ejection fraction. However, the anatomic position of coronary arteries differed between individual subjects, which could influence the myocardial infarction size, and consequently cause different deterioration of the left ventricular function even though the coronary artery was ligated at the same anatomic position.

The appropriate dosage for the angiogenesis effect of Tongxinluo in clinic is uncertain. We used two different dosages in this study, and the two dosages are similar to other animal studies. However, even if we use the low dosage in clinical practice, it would be equal to 7-14 times of the recommended clinical dosage. So whether regular recommended dose of Tongxinluo capsule has the same angiogenesis effect in coronary artery disease patients remains to be investigated. One pig in the highdose Tongxinluo group died before completing the study, and we noticed that this pig ate very little (which might due to the unpleasant flavor of Tongxinluo) and might die of malnutrition. However, we cannot exclude any toxic effects of the high dose Tongxinluo that finally cause the death of the pig.

Our study has two potential limitations. First, the sample size was relatively small. Second, Tongxinluo superfine powder was mixed with the food while breeding the laboratory animals. This would cause individual dose difference and lead to a pharmacodynamical variation among the study subjects. However, we tried to mix the study drug with less food and let the pigs eat all of them before breeding a full dose of the food. This would help to make sure that the pigs had eaten full dose of the study drug.

\section{Conclusion}

In a porcine model of AMI, traditional Chinese medicine Tongxinluo stimulates coronary collateral growth, which establishes Tongxinluo as an important complementary therapy for the treatment of AMI.

\section{Acknowledgments}

This study was supported by a grant from the Jiangsu Province Administration of traditional Chinese Medicine (200325), and a Project Funded by the Priority Academic Program Development of Jiangsu Higher Education Institutes (PAPD).

\section{References}

1. Roger VL, Go AS, Lloyd-Jones DM, Benjamin EJ, Berry JD, et al. (2012) Heart disease and stroke statistics--2012 update: a report from the American Heart Association. Circulation 125: e2-2e220.[Crossref]

2. Meier P, Gloekler S, Zbinden R (2007) Beneficial effect of recruitable collaterals: a 10 -year follow-up study in patients with stable coronary artery disease undergoing quantitative collateral measurements. Circulation 116: 975-983.

3. Schaper W (2012) Collateral vessels reduce mortality. Eur Heart $J$ 33: 564-566. [Crossref]

4. Wang W, Yang ZJ, Ma DC, Wang LS, Xu SL, et al. (2006) Induction of collateral artery growth and improvement of post-infarct heart function by hepatocyte growth factor gene transfer. Acta Pharmacol Sin 27: 555-560.[Crossref]

5. Lavu M, Gundewar S, Lefer DJ (2011) Gene therapy for ischemic heart disease. J Mol Cell Cardiol 50: 742-750.[Crossref]

6. Wu T, Harrison RA, Chen X (2006) Tongxinluo (Tong xinluo or Tong-xin-luo) capsule for unstable angina pectoris. Cochrane Database Syst Rev 18: CD004474.

7. Chen WQ, Zhong L, Zhang L (2009) Chinese medicine tongxinluo significantly lowers serum lipid levels and stabilizes vulnerable plaques in a rabbit model. $J$ Ethnopharmacol 124: 103-110.

8. Zhang L, Liu Y, Lu XT (2009) Traditional Chinese medication Tongxinluo dosedependently enhances stability of vulnerable plaques: a comparison with a high-dose simvastatin therapy. Am J Physiol Heart Circ Physiol 297: H2004-2014.

9. Bu PL, Zhao XQ, Wang LL (2008) Tong-xin-luo capsule inhibits left ventricular remodeling in spontaneously hypertensive rats by enhancing PPAR-gamma expression and suppressing NF-kappaB activity. Chin Med J (Engl) 121: 147-154.

10. Dai W, Wei C, Kong H (2011) Effect of the traditional Chinese medicine tongxinluo on endothelial dysfunction rats studied by using urinary metabonomics based on liquid chromatography-mass spectrometry. J Pharm Biomed Anal 56: 86-92.

11. Liang JQ, Wu K, Jia ZH (2011) Chinese medicine Tongxinluo modulates vascular endothelial function by inducing eNOS expression via the PI-3K/Akt/HIF-dependent signalingpathway. J Ethnopharmacol 133: 517-523.

12. Li C, Lossnitzer D, Katus HA (2012) Comparison of left ventricular volumes and ejection fraction by monoplane cineventriculography, unenhanced echocardiography and cardiac magnetic resonance imaging. The international journal of cardiovascular imaging 28: 1003-1010.

13. Rentrop KP, Cohen M, Blanke H (1985) Changes in collateral channel filling 
immediately after controlled coronary artery occlusion by an angioplasty balloon in human subjects. J Am Coll Cardiol 5: 587-592.

14. Chen XJ, Bian ZP, Lu S, Xu JD, Gu CR (2006) Cardiac protective effect of Astragalus on viral myocarditis mice: comparison with Perindopril. Am J Chin Med 34: 493-502. [Crossref]

15. Wu Y (2009) Collateral theory and vascular lesion treatment. Am J Chin Med 37: 241252.[Crossref]

16. Bai WW, Xing YF, Wang B (2013) Tongxinluo Improves Cardiac Function and Ameliorates Ventricular Remodeling in Mice Model of Myocardial Infarction through Enhancing Angiogenesis. Evidence-based complementary and alternative medicine 813247.

17. Hirota K, Semenza GL (2006) Regulation of angiogenesis by hypoxia-inducible factor 1. Crit Rev Oncol Hematol 59: 15-26.[Crossref]

18. Giacca M, Zacchigna S (2012) VEGF gene therapy: therapeutic angiogenesis in the clinic and beyond. Gene Ther 19: 622-629.

19. Kawasaki K, Smith RS, Hsieh CM (2003) Activation of the phosphatidylinosito 3-kinase/protein kinase Akt pathway mediates nitric oxide-induced endothelial cell migration and angiogenesis. Molecular and cellular biology 23: 5726-5737.

20. Dimmeler S, Fleming I, Fisslthaler B, Hermann C, Busse R, et al. (1999) Activation of nitric oxide synthase in endothelial cells by Akt-dependent phosphorylation. Nature 399: 601-605.[Crossref]
21. Zhao JL, Yang YJ, You SJ (2006) Effect of adenosine on endothelin-1 in the infarcted reflow and no-reflow myocardium of mini-swine. Acta Academiae Medicinae Sinicae 28: $225-229$.

22. Tonnessen T, Naess PA, Kirkeboen KA (1993) Release of endothelin from the porcine heart after short term coronary artery occlusion. Cardiovasc Res 27: 1482-1485.

23. Iglarz M, Clozel M (2007) Mechanisms of ET-1-induced endothelial dysfunction. $J$ Cardiovasc Pharmacol 50: 621-628.[Crossref]

24. Félétou M, Vanhoutte PM (2006) Endothelial dysfunction: a multifaceted disorder (The Wiggers Award Lecture). Am J Physiol Heart Circ Physiol 291: H985-1002.[Crossref]

25. Wu XC, Lai J, Wu XF (2011) Effect of chronic psychological stress on vascular endothelial dysfunction rats and intervention tongxinluo on it. Zhongguo Zhongxiyijiehezazhi 31: 680-683.

26. Habib GB, Heibig J, Forman SA (1991) Influence of coronary collateral vessels on myocardial infarct size in humans. Results of phase I thrombolysis in myocardial infarction (TIMI) trial. The TIMI Investigators. Circulation 83: 739-746.

27. Billinger M, Kloos P, Eberli FR (2002) Physiologically assessed coronary collateral flow and adverse cardiac ischemic events: a follow-up study in 403 patients with coronary artery disease. J Am Coll Cardiol 40:1545-1550.

28. Hansen JF (1989) Coronary collateral circulation: clinical significance and influence on survival in patients with coronary artery occlusion. Am Heart J 117: 290-295.

29. Regieli JJ, Jukema JW, Nathoe HM (2009) Coronary collaterals improve prognosis in patients with ischemic heart disease. Int J Cardiol 132: 257-262.

Copyright: $(2017$ Wang GF. This is an open-access article distributed under the terms of the Creative Commons Attribution License, which permits unrestricted use, distribution, and reproduction in any medium, provided the original author and source are credited. 